\title{
Selecting the best, without BCR signalling
}

B cells expressing high-affinity B cell receptors (BCRs) undergo selective proliferation and population expansion in the germinal centre (GC), giving rise to memory $\mathrm{B}$ cells and plasma cells. However, it is not clear how exactly the BCR controls this process. Reporting in Science, Khalil et al. now show that, surprisingly, most proliferating GC B cells do not undergo active BCR signalling, owing to increased phosphatase activity, but they regain responsiveness to BCR signals in the G2 phase of the cell cycle.

The authors found that GC $B$ cells have very low levels of tyrosine phosphorylation of the BCR proximal signalling molecules SYK and BLNK in response to BCR ligation compared with naive or non-GC B cells. However, GC B cells responded normally to signals that bypass the BCR (namely, PMA and ionomycin), indicating that the $\mathrm{B}$ cells were not defective. The reduced tyrosine phosphorylation was shown to be due to increased phosphatase activity. Indeed, inhibition of phosphatase activity rescued SYK and BLNK phosphorylation, as well as $\mathrm{Ca}^{2+}$ flux, which was minimal in BCR-stimulated GC B cells.
Further analysis showed that GC $B$ cells had higher levels of active SHP1 (SH2 domain-containing phosphatase 1) and SHIP1 (SH2 domaincontaining inositol $5^{\prime}$ phosphatase 1), which negatively regulate BCR signalling, than naive and non-GC B cells. In GC B cells, SHP1 and SHIP1 which are constitutively associated with the BCR in resting B cells - did not relocalize to the opposite pole of the cell following BCR ligation as they do in non-GC B cells. B cell-specific

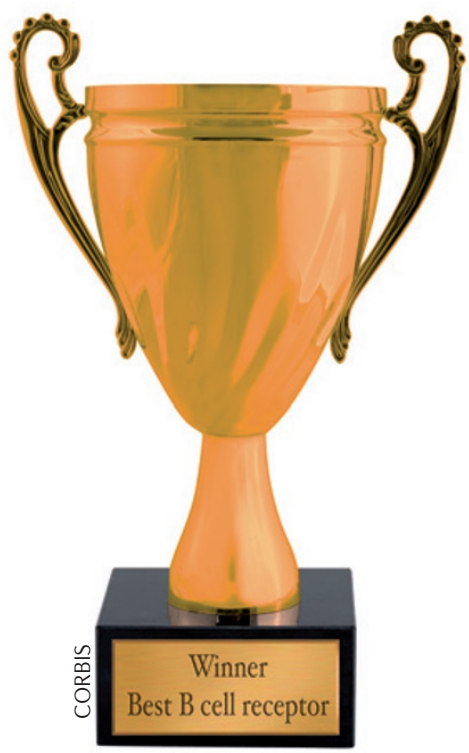

deletion of Shp1 resulted in a marked reduction in GC B cell frequencies, indicating the SHP1 is required for maintaining these cells.

Interestingly, a small fraction of BCR-stimulated GC B cells was found to generate phosphorylated SYK and relocalize SHP1 in response to BCR ligation. These cells were in the G2 phase of the cell cycle, suggesting that BCR signal transduction is cell cycle dependent.

The authors suggest that by linking BCR signalling to the cell cycle, GC B cells can integrate the dual functions of the BCR - antigen uptake for presentation to $\mathrm{T}$ helper cells and signalling. As BCR antigen capture is affinity dependent, $\mathrm{B}$ cells with high-affinity BCRs would outcompete other GC B cells for T celldependent survival and proliferation signals. But GC B cells would remain able to respond to BCR-derived signals to test the quality of new BCR mutants arising during the $S$ phase of the GC B cell proliferation cycle.

Olive Leavy

ORIGINAL RESEARCH PAPER Khali, A. M.,

Cambier, J. C. \& Shlomchik, M. J. B cell receptor signal transduction in the GC is short-circuited by high phosphatase activity. Science 3 May 2012 (doi:10.1126/science.1213368) 